Mon. Not. R. Astron. Soc. 000, 000-000 (0000) Printed 28 October $2018 \quad$ (MN LATEX style file v2.2)

\title{
Hydrodynamical Simulations of the Stream-Core Interaction in the Slow Merger of Massive Stars
}

\author{
N. Ivanova ${ }^{1}, \mathrm{Ph}$. Podsiadlowski ${ }^{1}$ and H. Spruit ${ }^{2}$ \\ 1 University of Oxford, Nuclear and Astrophysics Laboratory, Oxford, OX1 3RJ \\ 2 Max-Planck-Institut für Astrophysik, Karl-Schwarzschild-Strasse 1, 85741 Garching, Germany
}

28 October 2018

\begin{abstract}
We present detailed simulations of the interaction of a stream emanating from a masslosing secondary with the core of a massive supergiant in the slow merger of the two stars inside a common envelope. The dynamics of the stream can be divided into a ballistic phase, starting at the $L_{1}$ point, and a hydrodynamical phase where the stream interacts strongly with the core. Considering the merger of a 1 and $5 \mathrm{M}_{\odot}$ star with a $20 \mathrm{M}_{\odot}$ evolved supergiant, we present two-dimensional hydrodynamical simulations using the PROMETHEUS code to demonstrate how the penetration depth and post-impact conditions depend on the initial properties of stream material (e.g. entropy, angular momentum, stream width) and the properties of the core (e.g. density structure and rotation rate). Using these results, we present a fitting formula for the entropy generated in the stream-core interaction and a recipe for the determination of the penetration depth based on a modified Bernoulli integral.
\end{abstract}

Key words: binaries : close — hydrodynamics — nucleosynthesis

\section{INTRODUCTION}

A large fraction of massive stars are known to be members of binary systems which are close enough to interact strongly via Roche-lobe overflow (see e.g. Garmany, Conti \& Massey 1980). One of the most dramatic, but poorly understood types of binary interactions involves the complete merger of the two components in a common envelope which leads to the formation of a single, rapidly rotating star with possibly some highly unusual properties. This type of evolution generally occurs as the result of dynamical mass transfer (Paczyński \& Sienkiewicz 1972) where a more massive star (usually a giant) overfills its Roche lobe by an ever increasing amount leading to mass transfer on a dynamical time-scale. Since the companion is only able to accrete matter on its thermal time-scale, most of the excess matter is believed to form a common envelope engulfing both the mass donor and the secondary. Inside this envelope, the core of the giant and the secondary form a close binary. Friction between this immersed binary and the envelope then causes the orbit to shrink. If the energy released in the process is sufficient to eject the envelope, this leads to the formation of a very close binary consisting of the core of the giant and a largely unperturbed companion star (Paczyński 1976; Taam \& Sandqvist 2000). However, if the envelope is not ejected in the early rapid spiral-in phase, this phase is followed by a phase of selfregulated spiral-in where all the frictional energy deposited in the envelope can be transported to the surface where it is being radiated away (Meyer \& Meyer-Hofmeister 1979;
Podsiadlowski 2001). For a massive primary with a mass of $\sim 20 \mathrm{M}_{\odot}$ filling its Roche lobe as a red supergiant after the end of helium core burning, the characteristic time-scale for the self-regulated spiral-in phase is several $100 \mathrm{yr}$. However, at some point, the spiraling-in secondary itself will fill its critical tidal lobe and start to transfer mass to the core of the giant. This situation is in many respects similar to the case of normal mass transfer by Roche-lobe overflow except for several fundamental differences. (1) Mass transfer occurs inside a low-density, opaque envelope, where the density contrast between the envelope and the secondary's average density may be as high as 1:106. (2) The mechanism for driving mass transfer is orbital angular momentum loss due to the friction of the immersed binary with the envelope. Because of the short spiral-in time-scale this leads to extremely high mass transfer rates of order $0.01-10 \mathrm{M}_{\odot} \mathrm{yr}^{-1}$. (3) Since the spiral-in time-scale is much shorter than a typical synchronization time-scale in a radiative star (e.g. Zahn 1975), the spin of the secondary may not be synchronized with the orbit.

As a result of this interaction, the secondary is gradually being dissolved inside the common envelope. Even though the time-scale for this dissolution is short compared to the evolutionary time-scale of a giant, it is still much longer than the dynamical time-scale of the secondary. We therefore refer to this process as a 'slow merger' of the components, although this phase is likely to be terminated by a delayed dynamical instability when the flat-entropy core of 
the secondary is being exposed and mass transfer becomes catastrophic. This will ultimately lead to the dynamical disruption of the remnant core (if it is relatively unevolved).

In this paper we present the first part of a systematic study of the slow merger of massive stars, concentrating on the interaction of the mass stream emanating from the masslosing immersed star with the core of the giant. This stream will generally not intersect with itself and form an accretion disc, but will instead penetrate deep into the core of the giant before it is stopped by the increasing pressure inside the core. As we will show, the stream may be able to reach a radius as small as $\sim 10^{10} \mathrm{~cm}$. Since this is deep inside the hydrogen-exhausted helium core of a $20 \mathrm{M}_{\odot}$ star that has already exhausted helium in the core, this may have important consequences: (1) It can lead to the dredge-up of helium from the core which can then be mixed with the rest of the envelope. (2) Since the material in the stream is hydrogenrich and is heated to temperatures as high as several $10^{8} \mathrm{~K}$ in the post-impact region, this may lead to some unusual nucleosynthesis, in particular s-processing. How deep the stream can penetrate depends on the initial entropy of the stream material, but also its initial angular momentum and most importantly on the structure near the core and the amount of entropy generated in the interaction with the core. It is the purpose of this paper to systematically explore how the penetration depends on these various factors and to determine the properties of the post-impact material. In a subsequent paper, we will use these results to model the slow merger of two massive stars in the context of the progenitor of SN 1987A, whose highly anomalous properties are likely the result of such a merger (see Podsiadlowski 1992, 1997 for discussion and references). While the hydrodynamical simulations presented in this paper were designed with this application in mind, our analysis is sufficiently general to allow application to other systems that have been suggested to be post-merger objects (e.g. V Hydrae [Kahane et al. 1996]; FK Comae stars [e.g. Rucinski 1990; Welty \& Ramsey 1994]).

In Section 2 we outline the basic assumptions for modelling the initial properties of the stream leaving the masslosing secondary. In Section 3 we use this model to obtain a description of the stream at the start of the hydrodynamical simulations. Section 1 provides a description of the numerical method used in the hydrodynamical calculations. In Section 5 we present the results of detailed hydrodynamical simulations and systematically discuss the physics of the interaction between the jet and the ambient matter, in particular the mechanism of the stream dissipation, the effects of rotation and of nuclear burning in the stream. Finally, in Section 6 we present an semi-empirical prescription which allows to estimate the penetration depth, taking into account the entropy generated in the interaction.

\section{THE INITIAL CONDITIONS OF THE STREAM}

\subsection{Mass-loss rates}

Once the immersed secondary starts to fill its tidal lobe, it starts to transfer mass to the core of the giant. The mass-transfer rate is determined by the friction between the spiraling-in immersed binary and the envelope which drives the evolution of the system. Since the mass-loss time-scale is generally much shorter than the nuclear or thermal timescale for a main-sequence star, the radius responds adiabatically to mass loss, i.e.

$\frac{\dot{R}_{2}}{R_{2}}=\left(\frac{\partial \ln R_{2}}{\partial \ln M_{2}}\right)_{\text {ad }} \frac{\dot{M}_{2}}{M_{2}}=\zeta_{\text {ad }} \frac{\dot{M}_{2}}{M_{2}}$,

where $R_{2}$ and $M_{2}$ are the radius and the mass of the masslosing secondary, respectively.

The Roche-lobe radius $R_{\mathrm{RL}}$ of the secondary changes according to

$$
\begin{aligned}
\frac{\dot{R}_{\mathrm{RL}}}{R_{\mathrm{RL}}} & =\left(\frac{\partial \ln R_{\mathrm{RL}}}{\partial \ln M_{2}}\right)_{D=\mathrm{const}} \frac{\dot{M}_{2}}{M_{2}}+\left(\frac{\partial \ln R_{\mathrm{RL}}}{\partial t}\right)_{\dot{M}_{2}=0} \\
& \equiv \zeta_{\mathrm{RL}} \frac{\dot{M}_{2}}{M_{2}}+\left(\frac{\partial \ln D}{\partial t}\right)_{\dot{M}_{2}=0},
\end{aligned}
$$

where the Roche-lobe radius $R_{\mathrm{RL}}$ is given by Eggleton (1983)

$$
R_{\mathrm{RL}}=\frac{0.49 q^{2 / 3}}{0.6 q^{2 / 3}+\ln \left(1+q^{1 / 3}\right)} D .
$$

Here $q=M_{2} / M_{1}$ is the mass ratio, and $M_{1}$ is the total mass of the primary core within the binary separation $D$.

Assuming that the radius of the star is equal to the Roche-lobe radius, we can equate equations (1) and (2) and obtain an estimate for the mass-loss rate from the secondary as

$\frac{\dot{M}_{2}}{M_{2}}=\frac{1}{\zeta_{\text {ad }}-\zeta_{\mathrm{RL}}}\left(\frac{\partial \ln D}{\partial t}\right)_{\dot{M}_{2}=0}$,

where $\partial \ln D / \partial t$ is the change in the orbital separation due to tidal and viscous friction. For typical conditions in a $20 \mathrm{M}_{\odot}$ supergiant, the resulting mass-loss rates are typically in the range of 0.01 to $10 \mathrm{M}_{\odot} \mathrm{yr}^{-1}$ (Ivanova, Podsiadlowski \& Spruit 20001; and Ivanova \& Podsiadlowski 2002 [IP]). To obtain values for $\zeta_{\text {ad }}$, we performed separate stellarevolution calculations where mass was taken off the assumed secondary on a time-scale much shorter than the thermal time-scale in its outer envelope (see e.g. Podsiadlowski, Rappaport \& Pfahl 2001).

\subsection{The stream density profile and the stream width}

In previous treatments of mass outflow from $L_{1}$, it is generally assumed that the stream is isothermal (see e.g. Lubow \& Shu 1975; LS; Bisikalo et al. 1997). Because of the high expected mass-loss rate in our problem, it is more appropriate to assume that the stream behaves adiabatically, since the radiative diffusion time-scale is generally much longer than the characteristic flow time-scale (for a stream with a radius of $10^{10} \mathrm{~cm}$, the diffusion time is of order $10^{6} \mathrm{~s}$ ).

In the case where the secondary's spin is aligned and synchronous with the orbit, we can determine the density distribution in the neighbourhood of the $L_{1}$ point by expanding the potential around this point in a Taylor series (see e.g. LS), where we use Cartesian coordinates $(x, y, z)$ :

$\Phi=\Phi_{L 1}-(2 A+1) \frac{\left(x-x_{L 1}\right)^{2}}{2} \Omega^{2}+$ 


$$
A=\frac{\mu}{(A-1) \frac{y^{2}}{2} \Omega^{2}+A \frac{z^{2}}{2} \Omega^{2},},
$$

Here $\Omega$ is the angular speed of the binary and $\mu=$ $M_{1} /\left(M_{1}+M_{2}\right)$.

A natural assumption is that the energy distribution within the stream, consisting of kinetic and internal energies, follows the shape of the Roche potential and that the Bernoulli integral for the matter leaving the donor star is conserved (for an adiabatic stream)

$B \equiv \Phi+\frac{u^{2}}{2}+\frac{c^{2}}{\gamma-1}=\Phi_{L_{1}}+\frac{u_{L_{1} 2}}{2}+\frac{c_{L_{1}}^{2}}{\gamma-1}$.

Here $u_{L_{1}}$ and $c_{L_{1}}$ are the velocity and the sound speed of the matter at $L_{1}$, and $\gamma$ is the adiabatic index of the stream material. Let us assume that in the $L_{1}$ neighbourhood the stream is laterally in hydrostatic equilibrium. This is a good approximation, especially in the $Z$-direction (LS). Then, in the $Y Z$-plane

$\Phi-\Phi_{L 1}=\left(\frac{A-1}{2} y^{2}+\frac{A}{2} z^{2}\right) \Omega^{2} \approx \frac{\alpha}{2} r^{2} \Omega^{2}$,

where $r$ is the distance to the $L_{1}$ point and $\alpha \approx A$ is a unitless constant which depends on the shape of the potential in the neighbourhood of $L_{1}$. For binary systems with a mass ratio, $q$, between 0.006 and 1.0 , it has a value of 6.16 to 8.0 (LS). Then the density distribution within an adiabatic stream can be obtained from the condition of lateral hydrostatic equilibrium as

$\rho=\rho_{\mathrm{c}}\left(1-\frac{\alpha}{2} \frac{r^{2}}{\widetilde{W}^{2}}\right)^{1 /(\gamma-1)}$

where $\rho_{\mathrm{c}}$ is the density of the material in the core of the stream, and $\widetilde{W}$ is the characteristic width of the stream, which depends on the properties (e.g. entropy, temperature) of the matter in the secondary:

$\widetilde{W}=\sqrt{\frac{\gamma}{\gamma-1}} \frac{s^{\frac{1}{2}} \rho_{\mathrm{c}}^{1 / 3}}{\Omega}$.

Here $s=P / \rho^{\gamma}$ is the adiabatic constant of the material from the secondary. An adiabatic stream can not have a width wider than $W=\widetilde{W} \sqrt{2 / \alpha}$ (as can be seen from equ. 8 ).

We assume that the velocity of the material at $L_{1}$ is given by the local sound speed (see LS) and that this speed varies through the stream cross-section. Then, integrating the mass-flow rate across the stream cross section near $L_{1}$, we can relate the mass-loss rate $\dot{M}_{2}$ (in units of $\mathrm{M}_{\odot} \mathrm{yr}^{-1}$ ) to the density in the stream core for an adiabatic stream (with $\gamma=5 / 3)$ :

$$
\begin{aligned}
\rho_{\mathrm{c}}=1.4 \times 10^{-3} \mathrm{~g} \mathrm{~cm}^{-3} & \\
& \dot{M}_{2}^{1 / 2} \Omega_{4} s_{15}^{-3 / 4}\left(x_{\mathrm{s}}^{2}-\frac{\alpha}{2} x_{\mathrm{s}}^{4}+\frac{\alpha^{2}}{12} x_{\mathrm{s}}^{6}\right)^{-\frac{1}{2}},
\end{aligned}
$$

where $s_{15}=\left(P / \rho^{\gamma}\right) /\left(10^{15} \mathrm{cgs}\right)$ is the scaled adiabatic constant of the material from the secondary, $\Omega_{4}=\Omega / 10^{-4} \mathrm{~s}^{-1}$, $x_{\mathrm{s}}=W / \widetilde{W}$ is the unitless stream width.

When the stream forms in the hot and high-pressure environment of a common envelope, the actual stream width is determined by the local conditions of the envelope. If we assume that the outer edge of the stream is in pressure balance with the common envelope, the stream density at the edge of the stream cannot be less than

$\rho_{\text {out }}=\left(P_{\mathrm{CE}} / s\right)^{1 / \gamma}$,

where $P_{\mathrm{CE}}$ is the ambient pressure in the common envelope. The stream width for the current mass loss rate then can be found from the equ. (10) in combination with

$\rho_{\mathrm{c}}=\left(P_{\mathrm{CE}} / s\right)^{1 / \gamma}\left(1-\frac{\alpha}{2} x_{\mathrm{s}}^{2}\right)^{-1 /(\gamma-1)}$

(from equ. 8).

\subsection{The effect of non-synchronous rotation}

Since the secondary's spin may not be synchronous with the orbit (in fact, it may be rotating faster or more slowly depending on the circumstances), we also need to consider the case of non-synchronous rotation. For this purpose we adopt a coordinate system that rotates with the secondary. At the equipotential surface which determines the surface of the secondary (the donor), there is an area where matter is being accelerated outwards from the stellar surface. From this zone matter may flow out. In the case of a synchronized secondary, the physical situation is a close analogue of a Laval nozzle, where the equipotential surfaces take over the role of a nozzle. In the standard case, when a thin flow is considered, $L_{1}$ can be taken as a point. Then, as for the case of the standard Laval nozzle, matter is almost completely unaffected by external acceleration at the nozzle mouth (at the sonic point), but experiences negative acceleration before and positive acceleration after passing though the nozzle.

For an asynchronously rotating secondary, the position of the positive acceleration zone with respect to the secondary changes with time. To parametrize the degree of asynchronicity, we introduce a parameter $f$,

$f=\frac{\Omega_{\mathrm{sec}}}{\Omega}-1$,

where $\Omega_{\text {sec }}$ is the angular velocity of the secondary. This parameter is zero if the secondary rotates synchronously. The position of the point where the acceleration experienced by matter on the stellar surface becomes non-negative can be found similarly to the $L_{1}$ point (in order to avoid confusion with the standard $L_{1}$ point, we refer to this point as the 'leaving point', $L P$ ), considering the generalised Roche potential, $\Psi$, for the frame with the origin at the center of the secondary, which rotates with the secondary (see e.g. Limber 1963):

$\Psi=\Phi-\frac{\zeta^{2}+\eta^{2}}{2} f(f+2) \Omega^{2}$.

Here $\Phi$ is the usual potential of the binary

$\Phi=-\frac{G M_{1}}{\left|\boldsymbol{r}_{1}\right|}-\frac{G M_{2}}{\left|\boldsymbol{r}_{1}\right|}-\frac{1}{2}\left(\Omega \boldsymbol{e}_{z} \times \boldsymbol{r}\right)^{2}$

and $(\zeta, \eta)$ are Cartesian coordinates in the frame rotating with the secondary. For a secondary which rotates faster than the binary itself, $L P$ will normally be shifted from $L_{1}$ towards the donor; and for a secondary which rotates more slowly than the binary this point will be shifted towards the 
primary. A rough estimate of the linear speed with which the zone of positive acceleration travels across the secondary surface, $v_{\text {zone }}=f \Omega_{\mathrm{sec}} r_{\mathrm{sec}}$, shows that it is typically twice the local sound speed of the secondary (for the case of $f=1$ and considering parameters characteristic for a system at the point where the secondary starts to overfill its Roche lobe). In this case, we do not have a normal nozzle (as for a synchronized secondary), as matter at a particular position on the secondary's surface (in the frame rotating with the secondary) is not affected by any matter that has escaped from the secondary before it enters the zone of non-negative acceleration. Therefore we may consider what happens to a fluid element on the secondary independently from any matter that has flowed out previously. A physical analogue of this situation is a ball, containing the (secondary) matter, that is separated from the outside by negative acceleration. At the surface of the ball there is a hole which travels with supersonic speed. As far as the matter in the ball is concerned, the surface of the ball acts like a wall that is suddenly being removed (for some period of time) and then placed back again. Matter will escape from the ball through the hole only if the duration of the interval of positive acceleration is long enough for matter to be able to escape before it is pulled back by the negative acceleration. Another analogue of the problem is the sudden expansion of a gas sphere into a vacuum. In this case, the speed with which matter expands can be as high as 3 times the sonic speed (see e.g. Zeldovich \& Raizer 1966). In a real situation, we may expect a combination of these and other effects that cannot be easily treated analytically. As this discussion shows, for an a-synchronously rotating secondary, the range of the possible initial flow velocities includes significantly supersonic speeds. Fortunately, our ballistic calculations in $\S 3$ show that the stream properties close to the primary core are not strongly dependent on the initial flow velocity in the stream.

\subsection{The escape angle - generalization of the LS formalism}

In the case where the secondary's spin is not synchronous with the orbit, the angle at which matter leaves the secondary will be different from the synchronous case analyzed by LS. To obtain this angle in our problem, we generally follow the LS formalism with the following changes: we place the origin of the coordinate system not at $L_{1}$ but at LP, and we use a coordinate frame which rotates with the secondary. Using cylindrical coordinates, the potential in the neighbourhood of $L P$ can be expanded (in the $z=0$ plane) as

$$
\begin{aligned}
& \Phi=\Phi_{L P}-\left[1+f(f+2)+\frac{A_{L P}}{2}(3 \cos 2 \theta+1)\right] \frac{r^{2}}{2} \Omega^{2} \\
& A_{L P}=\frac{\mu}{\left|x_{L P} / D-1+\mu\right|^{3}}+\frac{1-\mu}{\left|x_{L P} / D+\mu\right|^{3}}
\end{aligned}
$$

where $X_{L P}$ is the distance between the center of mass and $L P$ and $\theta$ is the angle between the flow direction of the stream and the direction of the primary.

The escape angle of the stream in the frame rotating with the secondary can then be found, analogously to the derivation in LS, from the equation

$$
\begin{aligned}
& \cos ^{2} 2 \theta_{s}+\frac{8}{3} \cos 2 \theta_{s} \\
& \quad+\frac{16}{9 A_{L P}^{2}}\left(\frac{A_{L P}}{2}+1+f(f+2)-\frac{9 A_{L P}^{2}}{16}\right)=0
\end{aligned}
$$

For the case of a synchronously rotating secondary $(\mathrm{f}=0)$, this equation reduces to the solution for $\theta_{s}$ obtained by LS. The angle with which the stream will escape from $L P$ in the frame co-rotating with the binary is given by the transformation

$\tan \theta=\frac{f \Omega D\left(1-\mu+x_{L P} / D\right)+v_{e} \sin \theta_{s}}{v_{e} \cos \theta_{s}}$,

where $v_{e}$ is the velocity of the stream at the nozzle (sonic for a synchronously rotating secondary).

\section{THE BALLISTIC PHASE}

The motion of the stream can be naturally divided into two phases, a (quasi-)ballistic phase where the stream trajectory is not strongly affected by the interaction with the ambient matter, which can be treated more or less ballistically, and a hydrodynamical phase where the dynamics of the stream is dominated by the hydrodynamical interaction with the core. Considering these two phases separately helps to avoid numerical problems (due to the complexities near the $L_{1}$ point) at the start of the hydrodynamical calculation. However, it also introduces some uncertainty since matter in the inner region surrounding the primary core may not be in complete co-rotation with the orbit and will have a backward motion with respect to the co-rotating frame. Such relative rotation causes a force which reduces the interception angle between the falling stream and the normal direction to the core relative to the predictions from a purely ballistic trajectory (see also $\S 5.3 .1)$.

The equations of motion of the stream are

$u_{x} \frac{\partial u_{x}}{\partial x}+u_{y} \frac{\partial u_{x}}{\partial y}=-\frac{\partial \Phi}{\partial x}+2 u_{y} \Omega-\frac{1}{\rho} \frac{\partial P}{\partial l} \frac{u_{x}}{u}$
$u_{x} \frac{\partial u_{y}}{\partial x}+u_{y} \frac{\partial u_{y}}{\partial y}=-\frac{\partial \Phi}{\partial y}-2 u_{x} \Omega-\frac{1}{\rho} \frac{\partial P}{\partial l} \frac{u_{y}}{u}$

Here $u=\sqrt{u_{x}^{2}+u_{y}^{2}}$ is the magnitude of the central stream velocity, and $u_{x}, u_{y}$ are its components. The pressure gradient along the path $(1 / \rho) \partial P / \partial l$ is included for completeness. This term is generally small, since the Mach number of the flow is large except near the nozzle. It is kept here for completeness and consistency in the calculations. The structure of the common envelope enters into these equations through the pressure boundary condition at the stream edge, which is set equal to the ambient pressure in the envelope. The density within the stream is related to the pressure by the adiabatic constant of the secondary material $s=P / \rho^{\gamma}=$ const. Together with a specified mass-flow rate, this set of equations provides a complete set that completely describes the dynamics of the stream in this phase. 


\subsection{Initial conditions for the hydrodynamical calculations}

The initial conditions for the hydrodynamical simulations are taken directly from the parameters obtained from the ballistic calculations for a range of binary parameters. Specifically, we consider parameters representing the spiralin and subsequent merger of a secondary of 1 and $5 \mathrm{M}_{\odot}$ inside a $20 \mathrm{M}_{\odot}$ evolved supergiant which has completed helium burning in the core and has a core mass of $\sim 7 \mathrm{M}_{\odot}$. To model the secondaries, we first followed their evolution to the same age as the primary and then evolved them further, subjecting them to very fast mass loss to model their adiabatic response to mass loss. These calculations give the radii of the stars and their surface entropies as a function of current stellar mass.

\section{THE NUMERICAL METHOD}

\subsection{The hydrodynamics code}

For the hydrodynamical simulations of the stream-core interactions we use a code based on the PROMETHEUS hydrodynamical code (Fryxell, Müller \& Arnett 1989). This is an Eulerian code, which uses a second-order Godunov-type scheme to solve the hydrodynamical equations, the piecewise parabolic method (PPM) of Colella \& Woodward (1994). This code has been widely used by different groups (see e.g. Kercek, Hillebrandt \& Truran 1998; KHT), and we refer to these papers for a detailed description of the code and numerical tests. To make it applicable to our problem, we had to make a number of modifications to the original code, in particular to the treatment of the gravity field, the boundary conditions, to the equation of state and the hydrodynamical equations themselves (to take into account the effects of a frame co-rotating with the binary). Since the mass transfer occurs in an opaque environment, radiative losses are not important and have been neglected.

In the presence of a large pressure gradient at the coreenvelope interface and a correspondingly strong gravitational field, Godunov-type schemes produce intrinsic accelerations, which during a few dynamical time-scales $t_{\mathrm{d}}$ create significant outward motion (KHT). For a first-order Godunov method it is possible to find an analytical formula to modify the interface states. Then source terms (the gravitational field in our case) will be balanced by flux differences (LeVeque 1998). This is not possible for higherorder Godunov-type schemes, where each problem requires a separate treatment (KHT). To reduce the artificial acceleration in our case, we assume that the gravitational field can be considered as constant in time and that there is no self-gravity. Then, in each time step, we modify the interface values before applying the Riemann solver, by reducing them from both (left and right) interfaces by a pressure flux caused by gravity. Furthermore, we enforce the condition of hydrostatic equilibrium at the boundary (for ghost cells 1 )

1 Ghost cells are a few additional cells (four in the case of a PPM code) at each boundary of the computational domain, where the values at these cells are determined by the boundary conditions. in the direction of the gravity field. These modifications ensure that any initial model remains stable for arbitrarily long time.

\subsection{The model set-up}

We performed 2-dimensional calculations in polar cylindrical coordinates, where we usually used $300 \times 300$ grid points. In the case of very narrow streams, we increased the resolution to $600 \times 600$ grid points. We also carried out test calculations with grids of higher resolution to satisfy ourselves that further increasing the number of grid points does not significantly affect the results. As a general rule, we ensure that the inflowing stream contains at least 20 grid points in the azimuthal direction. We also carried out a few 3-dimensional calculations (in spherical coordinates) for the case of a symmetrical (non-inclined) stream in a non-rotating frame. The results of these calculations showed that there were no significant differences between the 2- and 3-dimensional calculations with the same initial parameters. In particular, the penetration depths of the stream were very similar in both cases.

\subsubsection{Ambient matter}

To model the region of interest for the stream-core interaction inside the common envelope, we adopt powerlaw distributions for the temperature and the pressure, i.e $T(r)=T\left(r_{0}\right)\left(r_{0} / r\right)^{\alpha_{T}}$ and $P(r)=P\left(r_{0}\right)\left(r_{0} / r\right)^{\alpha_{P}}$. We fitted these power laws to the structure in actual CE calculations (as described in detail in IP). Typical values for $\alpha_{T}$ and $\alpha_{P}$ are in the range $(1.3 ; 5.2) \div(0.8 ; 3.2)$, but can be as high as $(1.7 ; 7)$ (corresponding to a structure with an adiabatic index $\left.\gamma_{\mathrm{amb}} \approx 1.44\right)$ and reasonably describe the regions of the stellar models at the the evolutionary stage of interest (IP). For our calculations here, we adopted parameters $\left(\alpha_{T} ; \alpha_{P}\right)=(1.3 ; 5.2)$ and $(0.8 ; 3.2)$ for models representing a $20+1$ and a $20+5$ CE simulation, respectively. Throughout the domain of our calculations, we assume that the ambient matter has a constant composition, similar to the composition in the core region of the primary, mainly helium. In the full stellar models, hydrogen is exhausted below a radius of $4 \times 10^{10} \mathrm{~cm}$, and the hydrogen mass fraction increases to $\sim 0.1$ at the outer edge of the domain (at $7.5 \times 10^{10} \mathrm{~cm}$ ). We have tested that these differences do not affect the results appreciably. In this parametrized model, we assume that the ambient matter is initially in (quasi-)hydrostatic equilibrium; as the model is parametrized by the pressure and temperature gradient, the gravitational field is then defined implicitly by the initial pressure gradient of the frame and the initial density distribution. In calculations where nuclear burning is included, we use a nuclear reactions network with 27 isotopes, which includes all important reactions up to ${ }^{25} \mathrm{Mg}$, in particular all $\alpha$ reactions and the complete hot ( $\beta$-limited) CNO cycle. The reaction rates were taken from the Thielemann nuclear reactions library (provided by $\mathrm{R}$. C. Cannon) The changes in chemical composition and the energy density due to the nuclear burning are updated after each time step in the hydrodynamical calculation. To prevent nuclear burning in the ambient medium, we assumed that the ambient medium contained neither $\mathrm{H}$ nor $\mathrm{He}$ in 
these particular calculations. (For further details we refer to IP.)

\subsubsection{Boundary conditions}

The boundary conditions in the code, which describe the characteristics of the inflowing stream, are the internal and external Mach numbers, $M_{\text {int }}$ and $M_{\text {ext }}$; the ratio between the central density in the stream and the ambient density at the outer boundary $\eta_{\rho}$; the initial inclination angle of the stream $\theta$ (i.e. the angle between the initial flow direction and the direction of the primary). For the chemical composition of the gas in the stream we use the abundances of the secondary.

The boundary conditions for the rest of the box in the radial direction are outflow boundaries (except in the gas inflow zone) where the condition of hydrostatic equilibrium has been imposed for ghost cells. The boundary condition in the azimuthal direction also assumes outflow conditions (we used a grid which normally covers an angle $\pi$ ). As Courant number we used $N_{\mathrm{CFL}}=0.6 \div 0.8$. A further parameter is the angular velocity with which the coordinate system rotates (most calculations were performed in the frame rotating with the primary core).

\section{INTERACTION BETWEEN THE STREAM AND THE AMBIENT MATTER}

\subsection{The initial stream-core interaction: establishing a steady stream}

Figure 11 shows the initial interaction of a stream with a massive core and illustrates the development of a stationary stream. The parameters for this simulation are characteristic for a $1 \mathrm{M}_{\odot}$ star filling its Roche lobe inside the envelope of a very evolved $20 \mathrm{M}_{\odot}$ red supergiant (after helium core burning). The panels on the left show the hydrogen abundance, while the panels on the right show the divergence of the velocity field. The latter was chosen because it shows shock structures particularly clearly.

The top panels show the stream just before its 'impact' with the core ( $569 \mathrm{~s}$ after the beginning of the simulation), while the second set of panels is close to the point of impact (at $778 \mathrm{~s}$; note the bow shock in the right panels). Immediately after the impact, stream matter bounces off the core, where most of it is just reflected by the core and continues to move in the forward direction (i.e. counter-clockwise), but some of it is pushed backwards, attaining an angular velocity component opposite to the rotation of the core. As material that has been stopped by the core is pushed by material in the stream following from behind, it starts to flow up again, driving two powerful shocks on the front and back side of the stream into the envelopes. These shocks compress the stream significantly. Once they have left the domain of the calculation, the stream has attained essentially a stationary configuration, where the point of deepest penetration $\left(R \simeq 2 \times 10^{10} \mathrm{~cm}\right)$ and the stream shape no longer change significantly. The overall flow pattern also becomes moreor-less stationary, where all the matter leaving the stream in the core impact region flows up again, being vigorously mixed with helium from the core in the process.

\subsection{Mechanisms for stream dissipation}

The main objective of our calculation is to determine how deep the stream can penetrate into the core of a massive star rather than the initial transient behaviour. In the quasistationary situation this depends mainly on the entropy that is generated in the stream-core interaction.

\subsubsection{Pressure discontinuity and entropy generation}

While the stream is in lateral pressure balance with the ambient matter at an early stage of infall, it becomes increasingly unbalanced as the velocity increases and becomes significantly supersonic relative to the ambient medium. The interaction between the stream and the ambient matter due to the jump in pressure can be treated in a simplified way as a Riemann problem (see e.g. LeVeque et al. 1998), a solution of which is a combination of shock and rarefaction waves. In the case where the stream expands, the rarefaction wave, propagating into the stream material, does not change the stream entropy. However, compression of the stream by the ambient matter, in the form of shocks moving into the stream, generates entropy inside the shocks.

For a strong shock, where the pressure jump $P_{\mathrm{amb}} / P_{\mathrm{s}} \gg$ 4 , the coefficient of entropy change, i.e. the ratio of the entropy of the shocked stream material, $S_{\mathrm{ss}}$, to the initial entropy in the stream, $S_{\mathrm{s}}$, can be estimated as

$K_{\mathrm{S}}=S_{\mathrm{ss}} / S_{\mathrm{s}} \approx$ const $\cdot P_{\mathrm{amb}} / P_{\mathrm{s}}$

(Zeldovich1966).

In our case, we rather expect the development of weak shocks. Then an estimate for $K_{\mathrm{S}}$ can be written as

$K_{\mathrm{S}} \approx 1+\mathrm{const} \cdot \eta_{\rho}^{\gamma-1}\left(\frac{M_{\mathrm{int}}}{M_{\mathrm{ext}}}\right)^{2}\left(P_{\mathrm{amb}} / P_{\mathrm{s}}-1\right)^{3}$

(Zeldovich1966). The pressure jump $P_{\mathrm{amb}} / P_{\mathrm{s}}$ changes with the distance to the primary core. In the case of a power-law parametrisation of the ambient pressure, equation (22) predicts that models with smaller power-law indices will result in smaller pressure jumps for the same initial stream properties and hence smaller values for $K_{\mathrm{S}}$ and that this coefficient increases as the power-law index increases.

In Figure 2 we present some of the key characteristics of a stream with the same parameters as in Figure 1, once a more-or-less stationary flow pattern has been established. Note in the top two panels that the entropy in the core of the stream is initially constant and that all the entropy generation takes places in two strong shocks at the stream boundaries (clearly seen in the $\boldsymbol{\nabla} \cdot \boldsymbol{v}$ panel). All of the stream matter has to pass through the intersection of these shock structures, where it essentially attains its final entropy and becomes comparable to the entropy of the ambient material, at least in the case of the $\log P / \rho^{\gamma}$. For the entropy the difference between ambient and stream matter is largely caused by the difference in molecular weights and its subsequent change is due to mixing of these two media rather than dissipation. At the point of deepest penetration $\left(\sim 2 \times 10^{10} \mathrm{~cm}\right)$, the stream matter has a temperature of $6 \times 10^{7} \mathrm{~K}$ and a density of $\sim 3 \mathrm{~g} \mathrm{~cm}^{-3}$, high enough for nuclear burning (see $\S 5.4)$. 

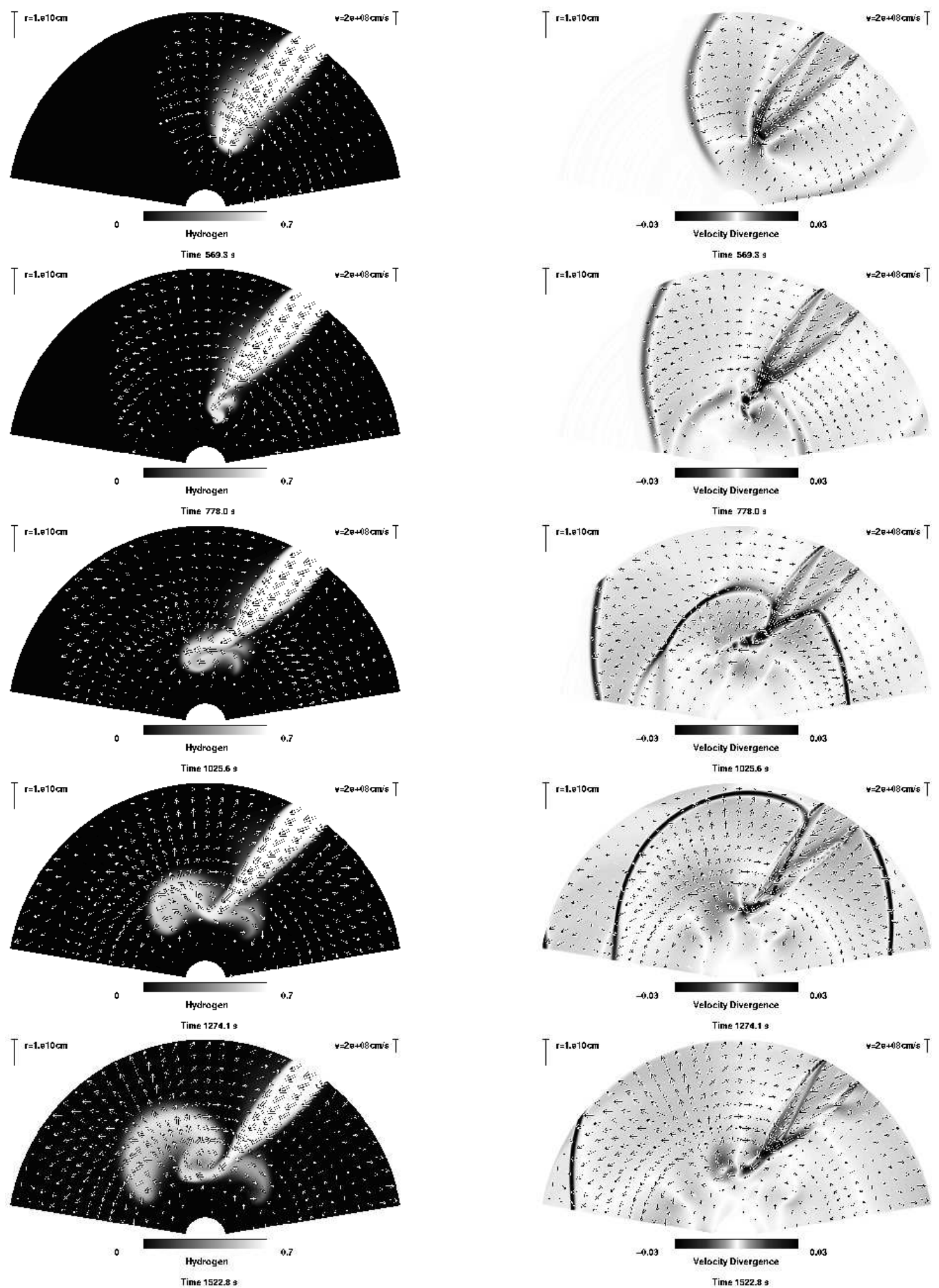

Figure 1. The initial stream-core interaction and the establishment of a steady stream in a frame rotating with the core (with an angular velocity $\Omega_{\text {frame }}=4 \times 10^{-4} \mathrm{rad} \mathrm{s}^{-1}$ ). The left panels show the distribution of hydrogen, the right panels the distribution of $\boldsymbol{\nabla} \cdot \boldsymbol{v}$. The initial Mach numbers in the stream, $M_{\text {int }}$, and ambient medium, $M_{\text {ext }}$ are 8.5 and 3.2 , respectively, the density ratio between the stream and the ambient medium, $\eta_{\rho}=8$, the outer radius $R_{\mathrm{T}}=7.5 \times 10^{10} \mathrm{~cm}$, the angular radius of the stream $R_{\mathrm{S}}=0.03 \pi$ and the initial stream inclination with respect to the radial direction $\Theta=13.5^{\circ}$. The velocity field is scaled according to $\log \left(v / 10^{6} \mathrm{~cm} \mathrm{~s} \mathrm{~s}^{-1}\right)$. 

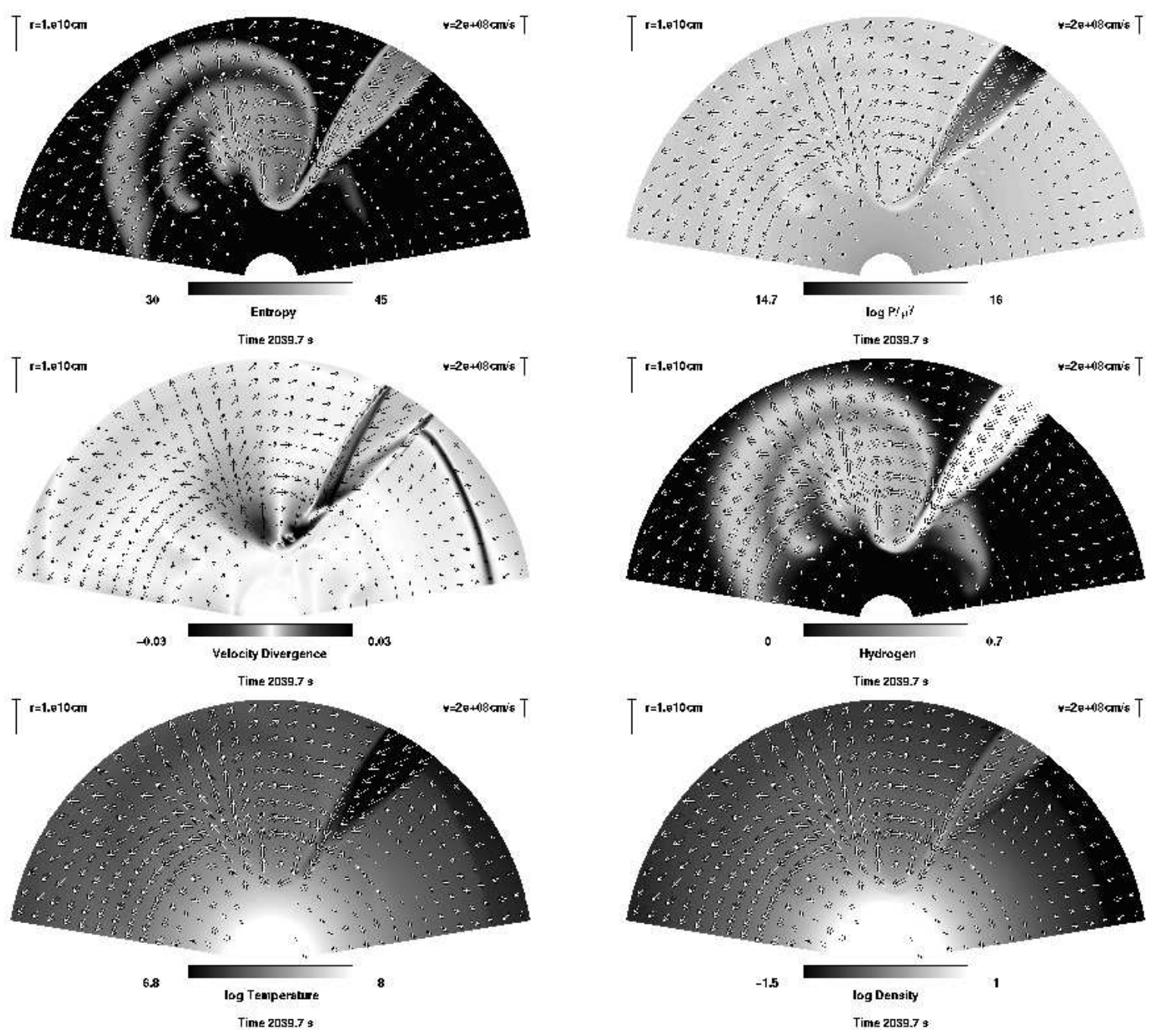

Figure 2. Characteristic properties (entropy, $\log P / \rho^{\gamma}$, divergence of velocity, hydrogen mass fraction, density and temperature) for a stationary inclined stream (with the same parameters as in Fig. If). The radius of deepest penetration is $2.0 \times 10^{10} \mathrm{~cm}$ in the simulation. At that depth the temperature in the stream is $6 \times 10^{7} \mathrm{~K}$ and the density $3 \mathrm{~g} \mathrm{~cm}^{-3}$.

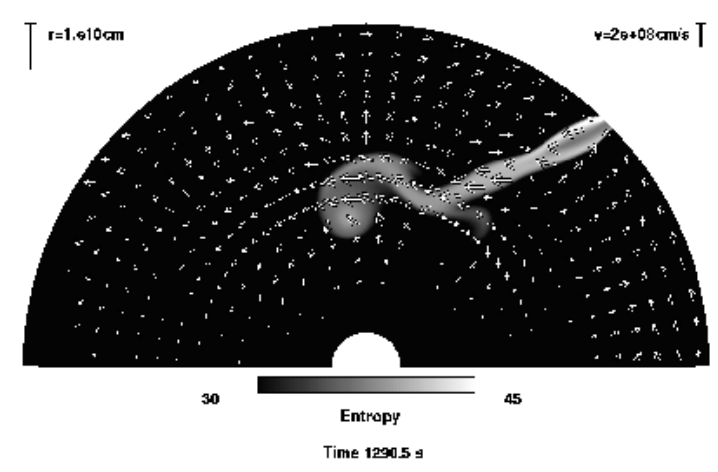

Figure 3. Example of a Kelvin-Helmholtz instability experienced by a narrow stream with $R_{\mathrm{S}}=0.005 \pi$. $\left(M_{\mathrm{int}}=8, M_{\text {ext }}=\right.$ $3.25, \eta_{\rho}=8, R_{\mathrm{T}}=7.5 \times 10^{10} \mathrm{~cm}, \Omega_{\text {frame }}=4 \times 10^{-4} \mathrm{rad} \mathrm{s}^{-1}$, $\Theta=18^{\circ}$.) The stream penetrates to a depth of $2.7 \times 10^{10} \mathrm{~cm}$.

\subsubsection{Kelvin-Helmholtz instabilities and the stream width}

The K-H instability affects both the stream shape and the stream width. For small relative velocities, the stream is "sausage"-shaped and becomes increasingly "snake"-shaped as the relative velocity increases (see Fig. 3). It becomes more important as the stream narrows, since perturbations of a given wavelength are more important for a narrower flow. It therefore determines the minimum stream width and thus limits the depth of penetration. In particular, a stream that is very narrow initially will penetrate less deep than a stream with a similar parameters like velocity, density and pressure, but with a larger mass-flow rate and hence larger initial stream width.

\subsection{The effects of rotation}

\subsubsection{A rotating medium}

In some cases, in particular near the end of the merging process, the envelope surrounding the spiraling-in components may no longer remain in co-rotation with the binary (either because the spiral-in time-scale becomes too short or the region surrounding the primary core expands rapidly). Then the stream feels an additional force due to the mov- 


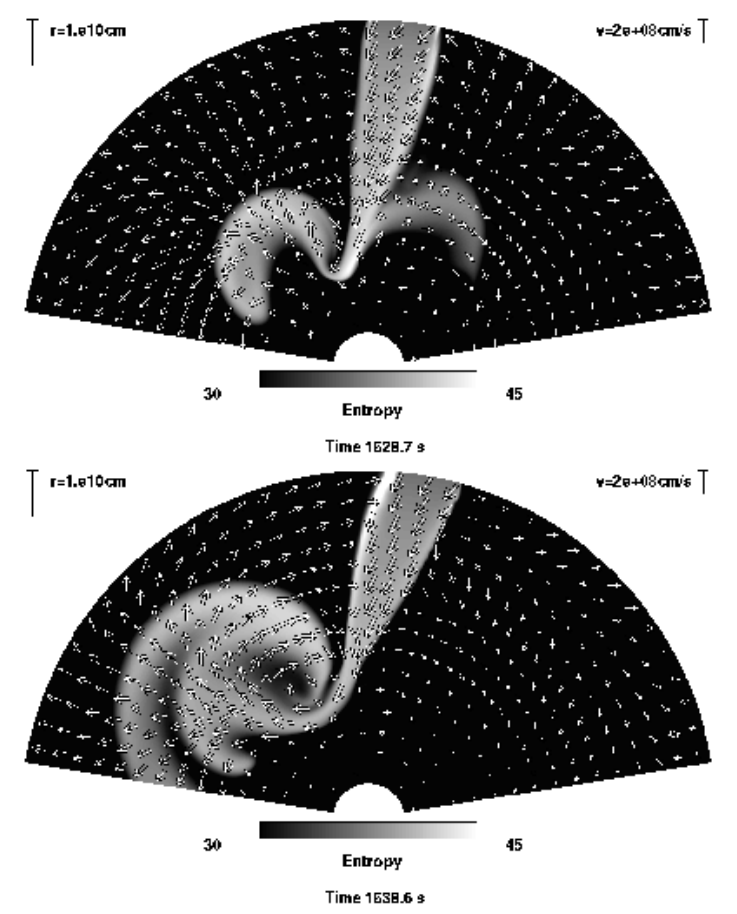

Figure 4. Examples for streams penetrating into a medium rotating relative to the binary. The stream rotates in an anticlockwise and a clockwise direction relative to the medium in the top and bottom panels, respectively, with a relative velocity $\Omega_{\text {shift }}=10^{-4} \mathrm{rad} \mathrm{s}^{-1}$. The initial positions of the stream centre in the azimuthal direction were taken to be $0.3 \pi$ and $0.6 \pi$ in the two cases, respectively. The other stream parameters are the same as in Figure日 $\left(M_{\mathrm{int}}=8.5, M_{\mathrm{ext}}=3.2, \eta_{\rho}=8, R_{\mathrm{T}}=7.5 \times 10^{10} \mathrm{~cm}\right.$, $\left.R_{\mathrm{s}}=0.03 \pi, \Omega_{\mathrm{amb}}=4 \times 10^{-4} \mathrm{rad} \mathrm{s}^{-1}, \Theta=13.5^{\circ}\right)$. The radii at the point of deepest penetration are 1.75 and $2.25 \times 10^{10} \mathrm{~cm}$, respectively.

ing external medium. This affects the stream's trajectory, in particular the angle of incidence, and thereby the penetration depth. This is illustrated in Figure 1 which shows two stream calculations with the same parameters as in Figure 2 but where the medium is assumed to rotate with an angular velocity $\Omega_{\text {shift }}=10^{-4} \mathrm{rad} \mathrm{s}^{-1}$ relative to the frame of the binary (rotating with angular velocity $\Omega$ ) either in the forward or in the backward direction. The frame of the medium (in which the calculation is performed) then rotates with a velocity $\Omega_{\mathrm{amb}}=\Omega \pm \Omega_{\text {shift }}$. In this frame, the stream itself rotates either anti-clockwise (top panel) or clockwise (bottom panel). As the stream is being pushed backwards by ambient matter moving against its direction of rotation, its trajectory steepens and consequently the penetration depth increases (top panel), while the opposite happens when the stream is pushed from behind (bottom panel). As a consequence the stream penetrates deeper to a radius of $\sim 1.75 \times 10^{10} \mathrm{~cm}$ instead of $\sim 2 \times 10^{10} \mathrm{~cm}$ in the standard case (Fig. 2), while it only reaches a depth of $\sim 2.25 \times 10^{10} \mathrm{~cm}$ in the opposite case. The corresponding change in the stream temperature at the deepest point is only a few per cent. Note also that for the stream rotating anti-clockwise, the entropy generation at the back side of the stream, where the dynamical pressure is smaller than at the front side (and hence has a larger jump in pressure), is larger than for stationary inclined stream.

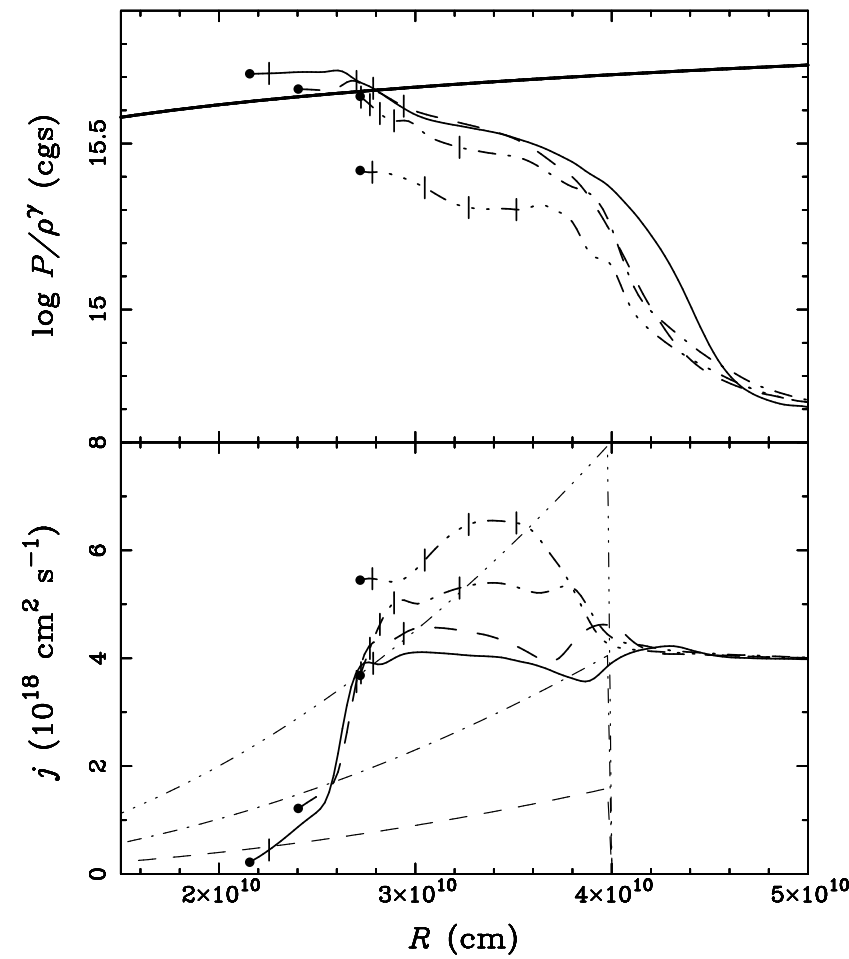

Figure 6. The logarithm of the adiabatic constant $\left(s=P / \rho^{\gamma}\right.$, top panel) and specific angular momentum (bottom panel) near the centre of the streams as a function of penetration depth for the simulations in Figure $\mathrm{f}$ with angular velocities $\Omega=0$ (solid curves), 0.001 (dashed curves), 0.0025 (dot-dashed curves) and $0.005 \mathrm{rad} \mathrm{s}^{-1}$ (dot-dot-dot-dashed curves). The corresponding angular-velocity profiles for the ambient core matter are shown in the bottom panel as lighter curves with the same line style. The thick solid curve in the top panel refers of $\log s$ in the ambient matter. The filled circles at the end of individual curves mark the points of deepest penetration. The ticks along each curve indicate particular values of the central stream hydrogen abundance by mass $(0.6,0.5,0.4,0.3$ from right to left).

\subsubsection{Core rotation}

As the stream interacts with the core, it injects not only matter but also angular momentum into the core, spinning it up in the process. Since the core only has to accrete a small fraction of the angular momentum available from the secondary to be spun up to critical rotation, we may expect that this itself will affect the dynamics of the interaction. To demonstrate this, we performed four simulations for streams with the same initial parameters and similar core properties except that we varied the rotation of the core from 0 to $0.005 \mathrm{rad} \mathrm{s}^{-1}$ within a core radius of $4 \times 10^{10} \mathrm{~cm}$. The results of these simulations are shown in Figures 5 and 6 .

As Figure 5 shows, the core-stream interaction changes qualitatively and quantitatively, where the penetration depth decreases with increasing core rotation. In the case of a rapidly rotating core, the penetration depth is centrifu-

2 Note that these cores are not completely comparable, since we assumed that the ambient core matter was in quasi-static dynamical equilibrium and had the same pressure and temperature distributions in all cases. This implies slightly different gravitational accelerations (and hence different core masses). 

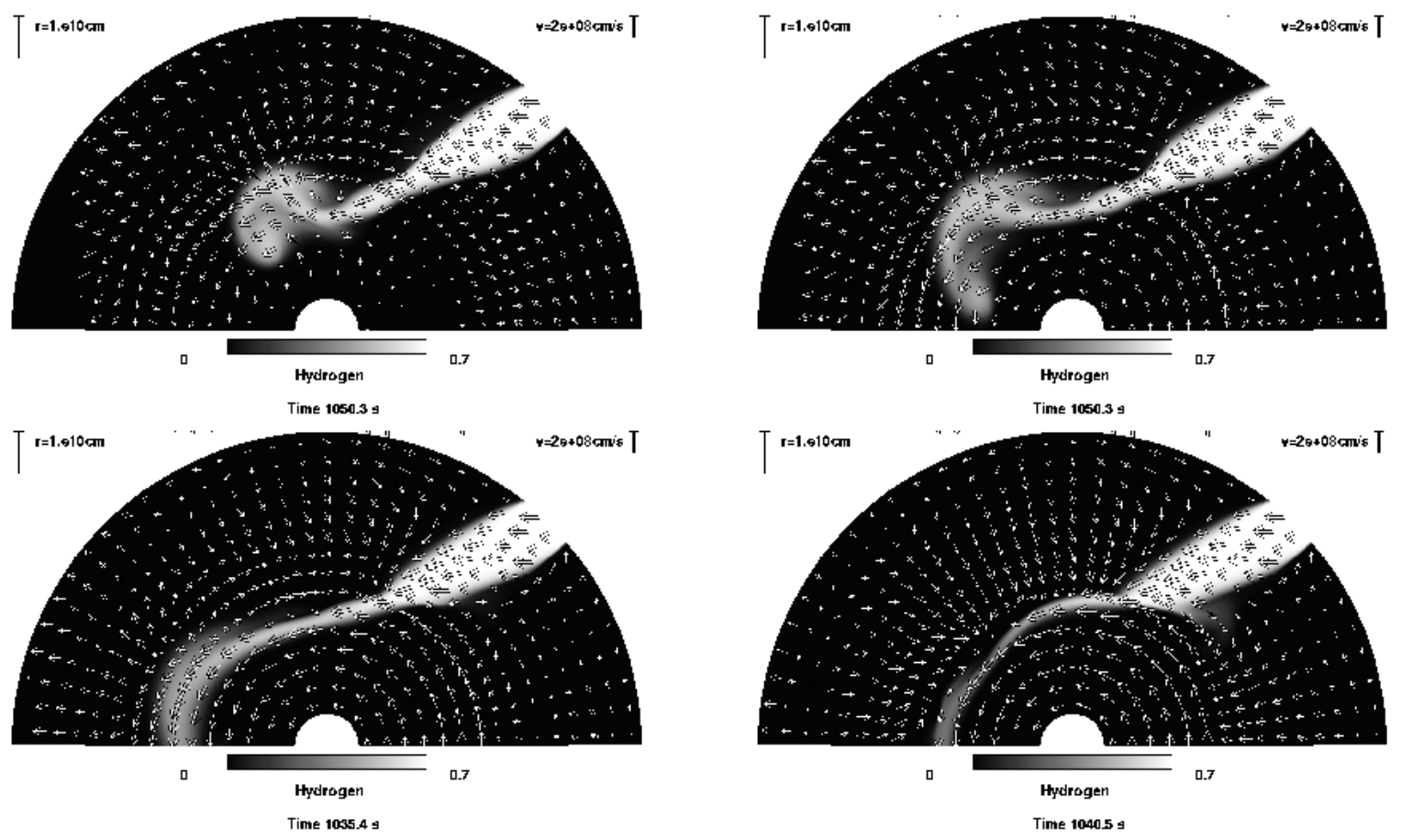

Figure 5. Stream simulations illustrating the effects of rotation of the primary core (in a frame rotating with the binary). In the four calculations, the core is assumed to rotate with constant angular velocities of $\Omega_{\text {core }}=0$ (top left), 0.001 (top right), 0.0025 (bottom left) and $0.005 \mathrm{rad} \mathrm{s}^{-1}$ (bottom right), respectively, up to a radius $4 \times 10^{10} \mathrm{~cm}$. The other stream parameters are $M_{\mathrm{int}}=9.8, M_{\mathrm{ext}}=3.7$, $\eta_{\rho}=8.4, R_{\mathrm{T}}=7.5^{10} \mathrm{~cm}, R_{\mathrm{s}}=0.025 \pi, \Theta=18^{\circ}$.

gally limited (i.e. by the angular momentum in the stream). In Figure 6 we show the logarithm of the adiabatic constant and the specific angular momentum near the center of the streams in the four simulations as a function of radius. In the two simulations with no or slow core rotation (solid and dashed curves), the stream's angular velocity is efficiently being braked below a radius $2.6 \times 10^{10} \mathrm{~cm}$ by its interaction with the core and reaches the deepest point of penetration (marked as filled circles) where the adiabatic constant is comparable to the ambient one. The specific angular momentum is always substantially less than the local critical angular momentum for centrifugal support, given by $\sqrt{g R^{3}}$, where $g$ is the gravitational acceleration at radius $R$. On the other hand, in the two cases with rapid core rotation (dot-dashed and dot-dot-dot-dashed curves), the stream is first accelerated in the azimuthal direction by the interaction with the core, in fact reaching an angular velocity that is larger than the local velocity of the core (even though the initial angular velocity at the core boundary was less). At the point of deepest penetration, the specific angular momentum is close to the critical angular momentum, and hence the stream becomes centrifugally supported. Note also that, in this case, the stream does not bounce but starts to form a ring orbiting the core and gradually mixing with it. This demonstrates that the main effect of rapid core rotation is to prevent/reduce the azimuthal braking of the stream. While this leads to less entropy generation in the stream (see the top panel in Fig. 6), which ordinarily would mean that the stream should be able to penetrate deeper, it makes the angular momentum of the stream itself a key factor in limiting the penetration depth. In the simulation with the fastest core, the stream matter is still substantially denser than the ambient matter at the final point shown in the simulations. Since it is also rotating faster than the local core material, it is reasonable to expect that the stream will continue to spiral in as it is being braked by the ambient medium and will penetrate somewhat deeper than is shown in the figures. (We were unable to follow the subsequent evolution since the stream matter was leaving the domain of the calculation at this point.)

Even in the case where the core is non-rotating, the specific angular momentum in the stream increases during a portion of its trajectory (see the solid curve in the bottom panel between 3 and $\left.3.8 \times 10^{10} \mathrm{~cm}\right)$. This is a direct consequence of the stream splitting into two components in the impact region, a forward and a backward component (this can be seen more clearly in Figs 1 and 2). As matter in the forward component is pushed by matter following from behind, it is being accelerated and gains angular momentum, while matter flowing backwards attains negative angular momentum.

Finally, note that because of the narrower stream in the post-impact region of the more rapidly rotating core, stream material mixes more efficiently with the core material (Fig. and 6). This may have been important consequences for the nucleosynthesis in this region (see $\S 5.4$ and IP).

\subsection{The effects of nuclear burning}

One of the objectives of our study is to investigate the nucleosynthesis in the stream material after it has been heated by its impact with the core of the primary. The associated heating by nuclear burning itself may then affect the hydrodynamics of the stream. As can already be seen from the 

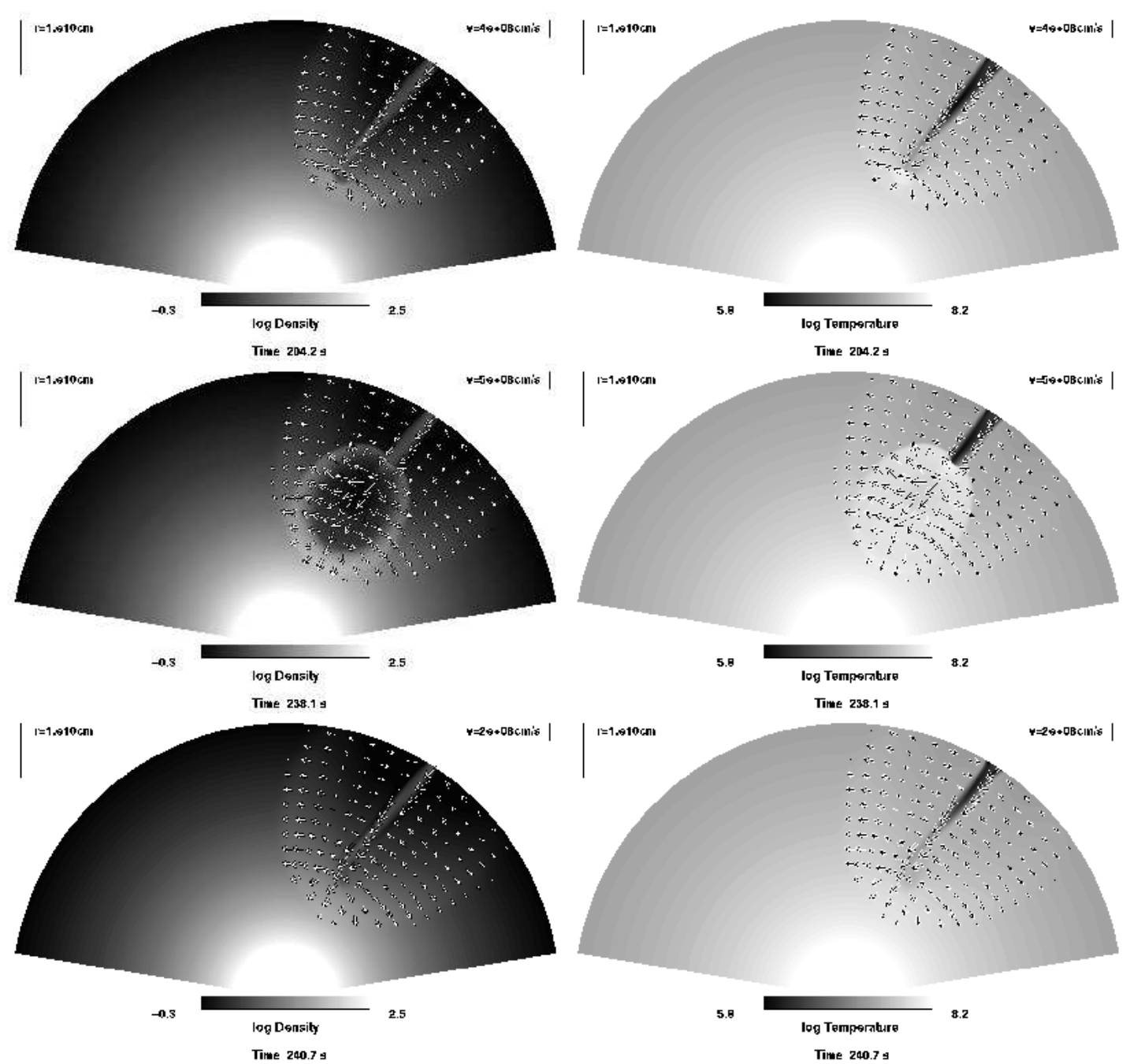

Figure 7. Density and temperature distributions for stream simulations illustrating the effect of violent nuclear ignition. The top two panels show the stream just before impact with the core and the point of nuclear ignition. The middle two panels show the detonation wave that develops shortly after nuclear ignition that disrupts the inflowing stream. The bottom two panels show a stream at the same time if nuclear burning is not included for comparison. The stream parameters are $M_{\text {int }}=9.9, M_{\mathrm{ext}}=4 ., \eta_{\rho}=7, R_{\mathrm{T}}=5 \times 10^{10} \mathrm{~cm}$, $R_{\mathrm{S}}=0.03 \pi, \Theta=10^{\circ}$.

simulations presented so far, the characteristic time-scale for the stream infall is of order $1000 \mathrm{~s}$, where the stream material typically spends no more than $50-100$ s near the impact region before it bounces or forms a ring orbiting the core (in the case of a fast rotating core). Nuclear burning can only affect the stream motion if the characteristic time-scale for nuclear burning is of order or shorter than the stream dynamical time-scale. For the largest part of the stream trajectory, the stream material is relatively cold (for the range of stream models considered).

Nuclear burning only starts to increase dramatically after it has been heated to a temperature comparable to the ambient temperature, which occurs suddenly in the region of impact. However, since the characteristic nuclear timescale is generally longer than the local dynamical time-scale, stream material will have already moved far away from the region of impact and hence have little effect on the stream dynamics (in the pre-impact region) and the stream trajectory. It mainly affects the properties of the ambient mat- ter (its composition and temperature) and hence the background conditions for the stream on a time-scale that is long compared to the stream infall time-scale (these effects and the associated nucleosynthesis can be followed with a standard stellar evolution code; see IPS for a description of the nucleosynthesis calculations).

The situation can be different when the stream impacts the core more-or-less radially and when the core has a very steep density gradient, causing a very strong shock and hence very high post-shock temperatures. This can cause a nuclear runaway as illustrated in Figure 1. As the middle panels in this figure show, it triggers a detonation wave that propagates up into the stream, almost completely disrupting it in the process. The expansion wave propagating into the ambient medium causes a dramatic expansion of the envelope material. Once all of the matter burning in this runaway has been consumed, one may expect that the stream re-establishes itself and that the process repeats, leading to pulsed nuclear burning. 


\section{THE PENETRATION DEPTH}

\subsection{The entropy change}

In the idealized case of a stream of constant entropy, the depth of penetration can easily be estimated from the balancing of the stream ram pressure with the ambient pressure. In our case, the pressure of the stream material becomes comparable to the ambient pressure well before the stream reaches its deepest point. In this case it is better to use an estimate based on the conservation of the Bernoulli integral for matter along a streamline (though both estimates would give similar results). However, since the stream entropy changes because of the compression by shock waves propagating into the stream from the sides, one has to use a modified Bernoulli integral to estimate the penetration depth. This is possible as long as the stream is wide enough that it is not strongly affected by the K-H instability and that core rotation plays a minor role.

For this purpose, we rewrite the Bernoulli integral for stream material in the form:

$\Phi_{\mathrm{T}}+\frac{u_{\mathrm{S}, \mathrm{T}}^{2}}{2}+\frac{c_{\mathrm{S}, \mathrm{T}}^{2}}{\gamma_{\mathrm{S}, \mathrm{T}}-1}=\Phi_{\mathrm{B}}+\frac{u_{\mathrm{S}, \mathrm{B}}^{2}}{2}+\frac{c_{\mathrm{S}, \mathrm{B}}^{2}}{\gamma_{\mathrm{S}, \mathrm{B}}-1}$

where $u$ is the velocity of stream material, $c$ the sonic speed of the stream material and $\Phi$ the potential. The subscript $\mathrm{S}$ corresponds to matter in the stream, the subscript $\mathrm{T}$ refers to the initial conditions and the subscript B to the conditions at the point of deepest penetration. The point of deepest penetration can then be determined by the condition that the pressure of the stream is equal to the pressure in the ambient matter and that the final stream velocity is zero (consistent with the results of our hydrodynamical calculations).

For the stellar structure parametrized by the pressure gradient the corresponding potential can be written as

$\Phi(r)=-\alpha_{\mathrm{P}} \frac{P(r)}{\rho(r)}=-\alpha_{\mathrm{P}} \frac{c_{\mathrm{A}}^{2}(r)}{\gamma_{\mathrm{A}}(r)}$,

where the subscript A corresponds to the ambient matter at the initial point of the stream. Assuming that the entropy of the stream changes according to

$$
\left(\frac{P}{\rho^{\gamma}}\right)_{\mathrm{S}, \mathrm{B}}=K_{\mathrm{s}}\left(\frac{P}{\rho^{\gamma}}\right)_{\mathrm{S}, \mathrm{T}},
$$

one can rewrite the Bernoulli integral to find $K_{\mathrm{s}}$ as a function of relative penetration depth, $\delta=r / r_{\mathrm{T}}$, and the initial conditions for the stream and ambient matter:

$$
\begin{aligned}
& -\frac{\alpha_{\mathrm{P}}}{\gamma_{\mathrm{A}}}+\frac{M_{\mathrm{ext}}^{2}}{2}+\frac{M_{\mathrm{ext}}^{2}}{M_{\mathrm{int}}^{2}} \frac{1}{\gamma_{\mathrm{S}, \mathrm{T}}-1}=-\frac{\alpha_{\mathrm{P}}}{\gamma_{\mathrm{A}}} \frac{\beta \mathrm{T}}{\beta(\delta)} \delta^{-\alpha_{\mathrm{T}}}+ \\
& \frac{1}{\gamma_{\mathrm{A}}} \frac{\gamma_{\mathrm{S}, \mathrm{B}}}{\gamma_{\mathrm{S}, \mathrm{B}}-1}\left(K_{\mathrm{s}} \eta_{\rho}^{1-\gamma_{\mathrm{S}, \mathrm{T}}} \rho_{\mathrm{A}}^{-\gamma_{\mathrm{S}, \mathrm{T}}} \frac{M_{\mathrm{ext}}^{2}}{M_{\mathrm{int}}^{2}} \frac{\gamma_{\mathrm{A}}}{\gamma_{\mathrm{S}, \mathrm{B}}}\right)^{\frac{1}{\gamma_{\mathrm{S}, \mathrm{B}}}} \rho_{\mathrm{A}} \delta^{\frac{1}{\gamma_{\mathrm{S}, \mathrm{B}}}-\alpha_{\mathrm{P}}} .
\end{aligned}
$$

Here $\rho_{\mathrm{A}}$ is the density of the ambient material at the initial radius of the steam, and $\beta$ is the ratio of gas pressure to total pressure for the ambient matter.

\subsection{Numerical fits}

To determine the entropy change factor, $K_{\mathrm{s}}$, we performed sets of hydrodynamical simulations for two parametrized

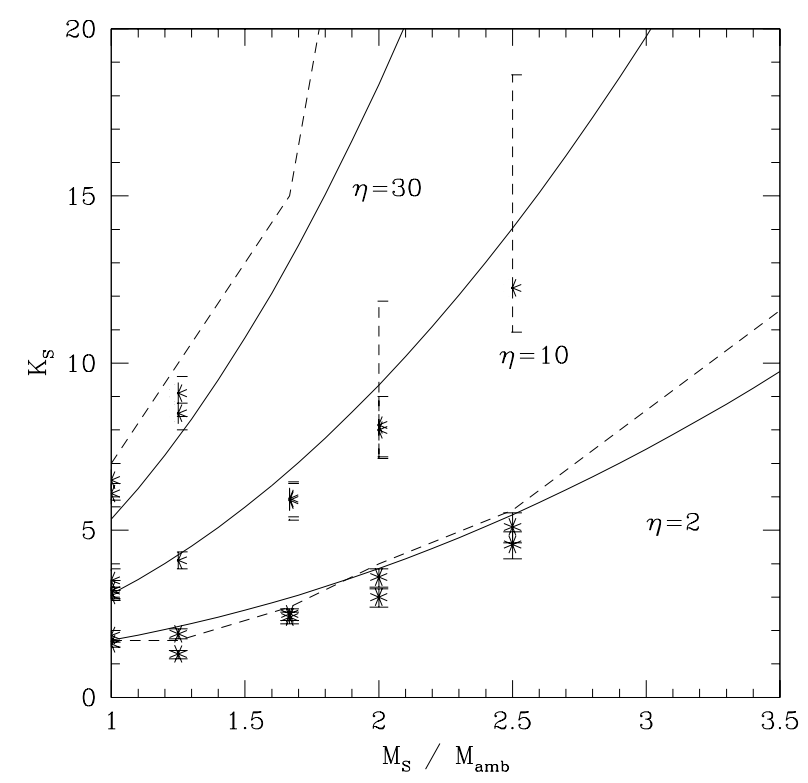

Figure 8. The entropy change as the function of the Mach numbers ratio for a parametrized ambient structure with $\alpha_{\mathrm{P}}=5.2$ and $\alpha_{\mathrm{T}}=1.3$. Asterisks correspond to values of $K_{\mathrm{S}}$ obtained from the hydrodynamical simulations by determining the appropriate value for $K_{\mathrm{S}}$ that would give the same penetration depth when using the Bernoulli integral as in the actual hydrodynamical simulation. The error bars are obtained by assuming an error in the determination of the penetration depth of 3 per cent. Solid curves correspond to the theoretical fit with $k=0.45$, while the dashed curves show the change in entropy of the stream material in the hydrodynamical simulations.

structures of the ambient medium, $\left(\alpha_{\mathrm{P}} ; \alpha_{\mathrm{T}}\right)=(5.2 .1 .3)$ and $(4.2 ; 0.9)$, representing typical profiles in massive CE systems. We then calculated the stream penetration depth for 3 values of density ratios, $\eta_{\rho}=2,10$ and 30, and for a number of models with different Mach number ratios, $M_{\text {int }} / M_{\text {ext }}$ (where we varied $M_{\text {int }}$, keeping $M_{\text {ext }}$ fixed). All streams were calculated with an initial stream inclination of $18^{\circ}$.

Using the expression for a weak shock (equ. 25), we fit the results to

$K_{\mathrm{s}}=1+k\left(\alpha_{\mathrm{P}}, \alpha_{\mathrm{T}}\right) \cdot \eta_{\rho}^{\gamma-1}\left(\frac{M_{\mathrm{int}}}{M_{\mathrm{ext}}}\right)^{2}$.

For an ambient medium, characterized by $\alpha_{\mathrm{P}}=5.2 ; \alpha_{\mathrm{T}}=$ 1.3 , these results are well fitted by a constant $k=0.45$ and for a structure with $\alpha_{\mathrm{P}}=4.2 ; \alpha_{\mathrm{T}}=0.9$ by $k=0.1$ (see Fig. 8).

\section{CONCLUSIONS}

We have investigated the physics of the stream interaction with the core of a massive star in the phase where a star immersed in a common envelope merges with the core and systematically explored how the penetration depth depends on the initial properties of the stream and the core structure. For simulations representing the merger of a 1 and $5 \mathrm{M}_{\odot}$ with an evolved $20 \mathrm{M}_{\odot}$ supergiant, it typically penetrates to a depth of $1-2 \times 10^{10} \mathrm{~cm}$, where it is being mixed vigorously with core material and is heated to temperatures 
$\gtrsim 10^{8} \mathrm{~K}$. In the case of a slowly rotating core, the penetration depth mainly depends on the initial entropy and the entropy that is generated in the interaction. This also depends on the initial width of the stream (which is mainly a function of the mass-transfer rate), since narrow streams are unstable to a Kelvin-Helmholtz instability. The main role of angular momentum in the stream is to determine the angle of incidence of the stream (which affects the dissipation in the stream). However, for a slowly rotating core, it is efficiently braked by the interaction with the core, spinning up the core in the process. In the case of a rapidly rotating core, the stream gains angular momentum from the core and the penetration is centrifugally limited. The stream behaviour is qualitatively and quantitatively different in the two cases. If the core is rotating slowly, the stream bounces off the core, quickly expanding away from the core, while in the case of a rapidly rotating core, the stream merges with the core in the form of a centrifugally supported ring.

In most cases, nuclear burning in the stream, which only becomes effective near the region of impact, does not affect the structure and dynamics of the stream itself, but affects the ambient medium which affects the long-term evolution of stream-core interaction. On the other hand, in the case of a very strong shock (in the case of a steep density gradient in the core and more or less normal incidence), shocktriggered nucleosynthesis can trigger a detonation wave that propagates upstream, temporarily disrupting the stream.

Using a simple model for the dissipation in a weak shock, we derived a simple recipe that allows the approximate determination of the penetration depth for a wide range of conditions. In a follow-up paper (IP), we will implement these results in a modified stellar-evolution code to model the whole merging phase of two stars in a slow merger.

\section{REFERENCES}

Bisikalo D.V., Boyarchuk A.A., Kuznetsov O.A., Chechetkin V.M., 1997, Astronomy Reports, 41 (issue 6), 794

Colella P., Woodward P.R., 1984, J. Comp. Phys. 54, 174

Eggleton P.P., 1983, ApJ 268, 368

Fryxell B.A., Müller E., Arnett W.D, 1989, Max-Planck-Institut für Astrophysik 449, Garching, Germany

Garmany C.P., Conti P.S., Massey P., 1980, ApJ, 242, 1063

Ivanova N., Podsiadlowski Ph., Spruit H., 2001, in Podsiadlowski Ph., Rappaport S., King A.R., D'Antona F., Burderi L., eds, Evolution of Binary and Multiple Star Systems, ASP Conf. Ser., Vol. 229, P. 261

Ivanova N., Podsiadlowski Ph., 2002 (IP), in preparation

Kahane C., Audinos P., Barnbaum C., Morris M., 1996, A\&A, 314,871

Kercek A., Hillebrandt W., Truran J.W., 1998, A\&A 337, 379

LeVeque R. J., 1998, J. Comp. Phys. 146, 346

LeVeque, R.J., Mihalas, D., Dorfi, E.A., Müller, E., 1998, in Steiner O., Gautschy A., eds, Computational Methods for Astrophysical Fluid Flow, Springer, Berlin

Limber D.N. 1963, ApJ 138, 1112

Lubow S.H., Shu F.U., 1975, ApJ 198, 383

Lubow S.H., Shu F.U., 1976, ApJ 207, L53

Meyer F., Meyer-Hofmeister E., 1979, A\&A, 78, 167

Paczyński B., 1976, in Eggleton P.P., Mitton S., Whelan J., eds, Structure and Evolution of Close Binaries. Kluwer, Dordrecht, p. 75
Paczyński B., Sienkiewicz R., 1972, Acta Astron., 22, 73

Podsiadlowski Ph., 1992, PASP, 104, 717

Podsiadlowski Ph., 1997, in SN 1987A: Ten Years After, CTIO/ESO/LCO Workshop, in press

Podsiadlowski Ph., 2001, in Podsiadlowski Ph., Rappaport S., King A.R., D'Antona F., Burderi L., eds, Evolution of Binary and Multiple Star Systems, ASP Conf. Ser., Vol. 229, P. 239

Podsiadlowski Ph., Rappaport S., Pfahl E., 2001, ApJ, in press astro-ph/0107261)

Rucinski S.M., 1990, PASP, 102, 306

Taam R.E., Sandquist E.L., 2000, ARA\&A, 38, 113

Welty A.D., Ramsey L.W., 1994, ApJ, 435, 848

Zahn J.-P., 1975, A\&A, 41, 329

Zeldovich Ya.B., Raizer Yu.P., 1966, Physics of Shock Waves and High-Temperature Hydrodynammics Phenomena, Academic Press, New York and London 